\title{
La Politique d'égalité femmes hommes 2014-2034 en Haïti : l'agentivité féministe, les possibilités institutionnelles et les contraintes récurrentes
}

\section{Haiti's Policy for Equality between Women and Men 2014-2034:} Feminist Agency, Institutional Opportunities and Recurrent Constraints

\section{La Política de igualdad entre mujeres y hombres en Haití : agentividad feminista, oportunidades institucionales y limitaciones recurrentes}

\author{
Stephen Baranyi et Hannah Champ
}

Volume 32, numéro 2, 2019

Militantisme et mobilisations

URI : https://id.erudit.org/iderudit/1068342ar

DOI : https://doi.org/10.7202/1068342ar

Aller au sommaire du numéro

Éditeur(s)

Revue Recherches féministes

ISSN

0838-4479 (imprimé)

1705-9240 (numérique)

Découvrir la revue

Citer cet article

Baranyi, S. \& Champ, H. (2019). La Politique d'égalité femmes hommes 2014-2034 en Haïti : l'agentivité féministe, les possibilités institutionnelles et les contraintes récurrentes. Recherches féministes, 32(2), 111-128.

https://doi.org/10.7202/1068342ar

\section{Résumé de l'article}

Selon certaines analystes, le mouvement des femmes, leurs voix et leurs priorités ont été largement déplacées par les agences internationales en Haïti, à la suite du séisme qui a frappé le pays en janvier 2010. Cette critique s’insère dans des analyses postcoloniales plus larges, de la marginalisation ou l'instrumentalisation des forces vives du Sud par des agences internationales, notamment dans les États qui se trouvent dans des situations fragiles ou en conflit. Sans minimiser la pertinence de ces critiques, la recherche menée entre 2017 et 2019 vise à comprendre la manière dont certains réseaux féministes résistent à ces dynamiques et influencent l'adoption de politiques publiques alternatives, particulièrement en Haïti. A été mobilisé un cadre théorique féministe institutionnaliste pour expliquer comment la militance du mouvement des femmes haïtiennes, l'intérêt de certains acteurs institutionnels et l'accompagnement de quelques agences internationales ont mené à l'adoption de la Politique d'égalité femmes hommes en 2014. Ce cadre théorique permet aussi d'expliquer la faible mise en oeuvre de cette politique et d'en tirer des leçons stratégiques, notamment par rapport à l'importance de l'agentivité hétérogène dans un contexte de résistances patriarcales et de crises récurrentes.
Tous droits réservés @ Recherches féministes, Université Laval, 2019
Ce document est protégé par la loi sur le droit d'auteur. L’utilisation des services d’Érudit (y compris la reproduction) est assujettie à sa politique d'utilisation que vous pouvez consulter en ligne.

https://apropos.erudit.org/fr/usagers/politique-dutilisation/ 


\section{La Politique d'égalité femmes hommes 2014-2034 en Haïti : l'agentivité féministe, les possibilités institutionnelles et les contraintes récurrentes ${ }^{1}$}

\section{STEPHEN BARANYI ET HANNAH CHAMP}

L'arrivée massive d'organisations humanitaires en Haïti à la suite du séisme le 12 janvier 2010 a contribué sans aucun doute à la réduction de la souffrance et éventuellement à la reconstruction du pays. Néanmoins, il est clair que cette marée humanitaire a aussi eu des retombées fort négatives, car elle a déplacé largement les institutions nationales de leur rôle d'entités directrices de la gouvernance et du développement national (République d'Haïti et OCDE 2011). En fait, la marginalisation des institutions étatiques et des associations a aggravé la tendance historique d'imposer sur le pays des schémas, des priorités et des projets avec peu d'ancrage local et donc peu d'avenir. Assistance mortelle, film du cinéaste haïtienaméricain Raoul Peck (2013), représente les effets destructeurs de l'aide internationale postséisme de façon dramatique.

L'expérience des organisations féministes dans ce contexte a été documentée par Denyse Côté (2014b). L'auteure démontre la manière dont le mouvement des femmes, leurs voix, leurs connaissances et leurs priorités ont été largement marginalisés par les agences humanitaires internationales après le séisme survenu en 2010. Elle explique comment les cadres de référence et les mécanismes de coordination adoptés par les agences internationales, notamment dans le cluster violence (espace de coordination sectoriel pour contrer la violence faite aux femmes) parrainé par le système onusien, ont peu pris en considération et parfois instrumentalisé les organisations haïtiennes qui travaillaient avec les femmes et les filles affectées par la violence depuis longue date.

Sans minimiser «la richesse et la complexité de la pensée féministe » et postcoloniale évoquée par Christine Verschuur (2010: 15), nous constatons que l'analyse de Côté renvoie aux critiques de la dépolitisation des organisations féministes par les agences internationales (Jad 2007), des interventions humanitaires thérapeutiques (McFalls 2010), des stratégies de contrôle biopolitique utilisées pour gérer l'instabilité dans le Sud (Duffield 2007) et des continuités coloniales qui, selon Célia Romulus (2018), sont à la base du discours sur l'État fragile haïtien.

Ces analyses postcoloniales sont importantes, car elles révèlent les contradictions des interventions internationales et leurs répercussions négatives sur

1 Le présent article est une version mise à jour de la communication présentée au Congrès international des recherches féministes dans la francophonie (CIRFF), à l'Université Paris Nanterre, le 29 août 2018. 
des sociétés du Sud. Par contre, elles souffrent parfois d'une diabolisation de l'international et d'une glorification des forces vives du Sud. Ce cadre théorique ne facilite donc pas toujours une fine compréhension de la diversité de ces institutions, ou des relations parfois constructives qui relient des mouvements féministes, des institutions étatiques et des agences internationales dans certains contextes du Sud.

Notre article explore ces zones d'ombre, à partir d'une étude de la Politique d'égalité femmes hommes 2014-2034 (Politique EFH) et du Plan d'action national d'égalité femmes hommes 2014-2020 (Plan d'action EFH) (République d'Haïti 2014b et 2014a). Nous mobilisons d'abord un cadre féministe institutionnaliste pour tracer la convergence des efforts des « fémocrates» (soit les fonctionnaires féministes) au sein de l'État, le plaidoyer des organisations féministes de la société civile et l'appui de certaines agences internationales qui ont contribué à ces acquis. Par la suite, nous utilisons ce cadre théorique pour analyser les contraintes qui expliquent la faible mise en œuvre des engagements et la pertinence d'actions collectives plus hétérogènes dans le contexte des crises récurrentes.

\section{Des débats théoriques et stratégiques}

Le féminisme institutionnaliste, apparemment peu connu dans les milieux féministes francophones, s'articule autour de plusieurs concepts clés. Si l'institutionnalisme traditionnel met l'accent sur la façon dont les institutions présentent des possibilités et des contraintes pour l'agentivité collective et le changement social, sa variante féministe insiste sur les dynamiques genrées de pouvoir qui traversent toutes les institutions (Mackay, Kenny et Chappell 2010; Mackay et Krook 2015). Sur cette base, certaines auteures se penchent sur les formes d'organisation et de représentation, les alliances et la synchronisation (timing) de l'action collective féministe qui produisent des changements (ou non) dans les politiques publiques (Mazur 2009). D'autres s'intéressent à l'agentivité des fémocrates au sein des États et à la manière dont elles mobilisent des répertoires de protestation pour confronter les résistances et les inégalités de pouvoir dans les États patriarcaux (Bereni et Revillard 2012). Certaines auteures mettent plutôt l'accent sur les synergies qui peuvent émerger entre les féministes de la société civile et leurs alliées à l'échelle de l'État national, ainsi que des agences internationales (Eyben et Turquet 2013).

Parallèlement à cette littérature largement occidentale, on voit l'émergence d'une littérature sur l'activisme féministe en Amérique latine et dans les Caraïbes qui, sans le reconnaître, mobilise aussi des concepts du féminisme institutionnaliste. Par exemple, l'ouvrage dirigé par Elizabeth Maier et Nathalie Lebon (2010) expose comment, malgré la diversité des formes d'organisation, certains mouvements ont construit des alliances intersectionnelles ${ }^{2}$ reliant des luttes de genre, de classe et de

2 Sur le concept et les pratiques intersectionnelles, voir Sirma Bilge (2010). 
LA POLITIQUE D'ÉGALITÉ FEMMES HOMMES 2014-2034 EN HAÏTI | 113

race, ainsi que des stratégies articulant le féminisme au sein et à l'extérieur de l'État. Dans un chapitre sur Haïti, Myriam Merlet (2010) documente la décision prise par le mouvement féministe, au cours des années 90, de s'investir dans l'espace des politiques publiques et des institutions statutaires. Cependant, compte tenu du mépris de l'État pour les droits des citoyens et des citoyennes, Merlet (2010 : 137-138; notre traduction) suggère ce qui suit :

L'action féministe doit s'articuler avec un vaste mouvement citoyen pour la défense des droits [...] Les organisations des femmes doivent travailler en synergie avec celles des étudiants, des syndicats et des activistes qui luttent pour $[\ldots]$ le droit à l'éducation, à la santé, à l'alimentation, à la vie dans un environnement sain.

Pour Nathalie Lebon (2010), la reconnaissance de nouvelles formes d'activisme et la capacité d'articuler différents courants féministes sont des clefs de voûte dans le contexte de résistances patriarcales. Elizabeth Maier (2010 : 344; notre traduction) renchérit en proposant la construction de «synergies entre les activistes, les théoriciennes et les fonctionnaires internationales", ainsi que la mobilisation créative de la « dialectique de l'agentivité nationale, régionale et internationale ».

Ces enjeux sont repris dans l'ouvrage dirigé par Gabrielle J. Hosein et Jane Parpart (2016), sur les stratégies féministes et leurs répercussions dans les pays anglophones des Caraïbes. Deborah McFee (2016) y mobilise des concepts institutionnalistes (telles les stratégies des coalitions politiques et leur capacité de s'adapter aux possibilités institutionnelles) pour comparer le cas de la Dominique avec celui de Trinité-et-Tobago. En ce qui concerne la Dominique, McFee explique comment le Bureau des affaires des femmes a impliqué le mouvement des femmes, ainsi que l'Église catholique et une association d'hommes, dans la formulation d'une politique qui a été adoptée à un moment opportun sous un gouvernement favorable en 2006 et mise en œuvre avec certains succès depuis lors. Par contre, du côté de Trinitéet-Tobago, le processus a été lancé par les organisations féministes de la société civile : certes, elles ont réussi à obtenir l'appui du gouvernement pour un texte plus radical, mais elles ont été abandonnées par les partis politiques devant l'opposition des églises et des groupements masculins. La leçon stratégique que l'auteure en retient est qu'une politique nationale de genre «ne peut pas être basée sur une notion idéalisée [...] de l'égalité des genres; plutôt, elle doit répondre aux limites et aux débats internes spécifiques, sur le genre, dans des sociétés particulières » (McFee 2016 : 126; notre traduction).

Dans le même ouvrage, Maziki Thame et Dhanaraj Thakur (2016) présentent le cas jamaïcain depuis une perspective théorique féministe socialiste. Elles montrent que le Bureau des affaires des femmes a organisé des consultations auxquelles ont participé les partis politiques dominants, ce qui a abouti à l'adoption de la politique en 2011. Elles notent certains progrès depuis lors, par exemple la création d'un réseau 
de 109 points focaux «genre» qui traverse le secteur public et qui assure le suivi partiel de la politique. Par contre, cette politique "protège le patriarcat, l'hétérosexisme et le statu quo, en faisant place aux femmes de la classe moyenne [...] dans l'État jamaïcain » (Thame et Thakur 2016 : 160; notre traduction).

Le débat théorique implicite entre McFee, Thame et Thakur, sur les possibilités et les limites des stratégies réformistes, trouve des échos dans la littérature sur l'activisme féministe en Haïti. Côté (2014a: 210) mobilise des concepts féministes institutionnalistes de manière implicite dans un article à caractère historique, en soulignant le «génie stratégique» d'un mouvement qui s'est reconstitué au fil des luttes démocratiques des années 80 et qui a construit un réseau d'organisations de la société civile comme Kay Fanm et Solidarite Fanm Ayisyèn (SOFA), consacrées aux interventions sur le terrain et au plaidoyer pour réformer les politiques publiques. Côté précise que ces organisations ont profité de l'évolution des normes internationales et d'occasions conjoncturelles pour obtenir la création du ministère à la Condition féminine et aux Droits des femmes (MCFDF) en 1994, la loi criminalisant le viol en 2005 et la codification du quota minimal de $30 \%$ dans la loi électorale en 2006. Cette auteure signale aussi la renaissance du mouvement malgré les énormes pertes occasionnées par le séisme en 2010, avec l'élaboration du second plan national de lutte contre les violences faites aux femmes.

Cette histoire est reprise dans un nouvel ouvrage sur l'activisme féministe en Haïti, dirigé par Sabine Lamour, Denyse Côté et Darline Alexis (2018). Plusieurs chapitres abordent les récentes tentatives de réforme. Sur la base d'une enquête auprès de 25 cadres supérieurs et cadres supérieures dans plusieurs ministères de 2013 à 2015, Rose Esther Sincimat Fleurant (2018 : 157) suggère que, en dépit des percées normatives, l'application de l'approche genre « reste au stade macro dans l'administration publique et est circonscrite dans des discours de circonstance au cours de certaines activités conjoncturelles » comme la célébration annuelle du 8 mars. L'auteure, qui a été elle-même cadre supérieure (senior) au MCFDF, attribue cet écart aux capacités et aux ressources limitées des bureaucrates pour consolider la « routinisation » de l'approche genre devant les résistances au sein de l'administration publique, elles-mêmes ancrées dans des dynamiques culturelles et structurelles plus vastes.

Ketleine Charles (2018) tire des conclusions similaires au sujet de la faible mise en œuvre du quota minimal de $30 \%$ pour la représentation des femmes dans les institutions politiques. Pour sa part, Pauline Lecarpentier (2018) fait valoir qu'un projet de loi sur la paternité responsable, proposé par une ministre activiste, a été dilué en raison de la résistance de parlementaires hommes et que les organisations féministes (statutaires et sociales) ont été marginalisées à cette étape et ont reçu peu d'appui pour assurer le suivi de cette loi. Danièle Magloire (2018 : 203), quant à elle, complète le panorama avec une analyse de l'antiféminisme en Haïti, à cause d'une mouvance qui cherche à « disqualifier la parole féministe, [à] la juguler, [à] la rendre inaudible ». Cette mouvance est portée par certaines églises et par des institutions 
LA POLITIQUE D’ÉGALITÉ FEMMES HOMMES 2014-2034 EN HAÏTI | 115

statutaires, notamment des partis politiques et le système judiciaire, où le masculinisme reste fort. Nous reviendrons à ces analyses pointues dans les prochaines sections de notre article.

\section{Le positionnement théorique et méthodologique}

Notre survol de la littérature suggère que, même si peu d'auteures du Sud se situent théoriquement dans le courant féministe institutionnaliste, certains concepts de cette école sous-tendent leurs analyses. C'est le cas notamment de l'agentivité des réseaux féministes reliant les institutions statutaires, les associations et les partenaires internationaux, des stratégies qui leur permettent de créer ou de profiter de possibilités institutionnelles et des contraintes institutionnelles, structurelles ou culturelles qui bloquent les changements dans certains contextes. Notre recensement met aussi en relief la contribution des auteures du Sud, par exemple l'attention qu'elles accordent aux résistances bureaucratiques, au masculinisme plus large et à l'importance des alliances hétérogènes pour faire face à ces résistances. Sans minimiser les débats entre divers courants théoriques féministes, nous voulons illustrer ici l'utilité d'un cadre féministe institutionnaliste en l'appliquant à l'étude des processus qui ont mené à l'adoption et à la mise en œuvre mitigée de la Politique EFH et du Plan d'action EFH en Haïti.

Notre étude de cas est ancrée dans une large recherche documentaire comportant des entretiens avec un échantillon de 19 intervenantes clés haïtiennes et canadiennes, menés par Hannah Champ en 2017. Elle repose aussi sur la recherche menée par Stephen Baranyi et d'autres auteurs en Haïti depuis 2006, notamment sur la construction de la paix (Baranyi 2008) et sur les personnes vivant en situation de handicap (Baranyi et Louis 2016). Finalement, elle a été enrichie par des entretiens effectués par Baranyi avec le milieu associatif en juin et en juillet 2018, ainsi que par sa collaboration continue avec le MCFDF et des dirigeantes du mouvement féministe dans le contexte d'un projet financé par Affaires mondiales Canada ${ }^{3}$.

Pleinement conscients de notre positionnalité en tant que chercheur et chercheuse d'origine canadienne et blancs, nous ne prétendons pas parler au nom des Haïtiennes. Ce droit de parole reste entièrement le leur. À cet égard, nous remercions nos collègues haïtiennes qui ont généreusement partagé leurs connaissances avec nous au fil des années et nous espérons que notre analyse alimentera leurs réflexions et leur lutte sans répit pour la transformation d'Haïti.

3 Le projet AMC/PSOPS 17-043 avait pour titre «Renforcement des capacités institutionnelles pénales et sécuritaires haïtiennes ». 


\section{La construction sociale de la Politique EFH}

Une fois qu'elles s'étaient réorganisées après le séisme en janvier 2010, les fémocrates au sein de l'État et les militantes du mouvement historique des Haïtiennes ont choisi prioritairement de relancer leur plaidoyer au niveau politique. Sachant que le nouveau président et le Parlement considéraient des modifications à la Constitution de 1987, elles ont profité de cette occasion afin de demander le renforcement du quota minimal de $30 \%$ pour la représentation des femmes dans les institutions publiques. Par l'entremise d'un plaidoyer public provenant des organisations féministes et de conversations plus discrètes au sein de l'exécutif et avec le Parlement, menées par la ministre de la Culture et de la Communication à l'époque (et ancienne ministre à la Condition féminine et aux Droits des femmes), les fémocrates et les militantes ont plaidé pour l'inclusion du quota minimal dans la loi mère modifiée (Dumont 2017; Champ 2018). À la suite de ce plaidoyer coordonné, le président a approuvé en mai 2011 une série de modifications à la Constitution, y compris l'article 17.1 qui applique le quota minimal de $30 \%$ ainsi que l'article 31.1 qui renforce l'application de ce principe au sein des partis politiques.

C'est aussi dans cette conjoncture que le MCFDF et ses partenaires ont terminé le Diagnostic des inégalités de genre lancé en 2009. Sur la base du travail antérieur, notamment le premier rapport sur le suivi de la Convention pour l'élimination de toutes les formes de discriminations à l'égard des femmes en Haïti (CEDEF) (MCFDF 2008), le MCFDF a instauré la mise à jour participative du Diagnostic en 2011, dans les dix départements du pays. ONU-Femmes et l'Agence espagnole de coopération au développement ont appuyé ce travail aux niveaux technique et financier (MCFDF 2013). Les consultations ont alimenté la finalisation du Diagnostic et préparé le terrain pour l'élaboration de la Politique EFH.

Tout en reconnaissant les efforts du MCFDF et les acquis, tels que la ratification de la CEDEF en 1996 et l'établissement de la Concertation nationale contre les violences faites aux femmes en 2003, le Diagnostic a démontré « qu'Haïti continue de faire face à d'énormes problèmes d'inégalités liés aux relations sociales discriminatoires établies entre les femmes et les hommes dans tous les champs d'action » (MCFDF 2013 : 12). Le rapport a documenté ces discriminations et ces inégalités sur le plan symbolique et matériel, notamment dans les domaines de l'éducation, de la justice, de la santé, de l'emploi et de la participation politique. Sur cette base, le Diagnostic a proposé un ensemble de recommandations dans chaque domaine.

Selon des informatrices de l'ancienne Agence canadienne de développement international (ACDI), l'élaboration du Diagnostic a permis de constater que le MCFDF et les associations féministes haïtiennes avaient la capacité de terminer l'état des lieux et d'élaborer les grandes lignes des politiques alternatives, mais pas nécessairement de les convertir en outils de gestion axée sur les résultats (Champ 2018). Profitant des liens de coopération déjà établis, le MCFDF a sollicité et obtenu 
LA POLITIQUE D'ÉGALITÉ FEMMES HOMMES 2014-2034 EN HAÏTI | 117

l'appui de l'ACDI et du ministère des Relations internationales du Québec pour approfondir ce travail, par l'intermédiaire du Projet d'appui au renforcement de la gestion publique en Haïti (PARGEP) et du Projet d'appui technique en Haïti (PATH), dont les objectifs étaient de renforcer un éventail de capacités techniques du MCFDF et d'autres ministères.

Comptant sur le soutien des partenaires techniques et financiers (PTF) internationaux clés, les cadres du MCFDF ont dirigé un groupe de travail interinstitutionnel (réunissant notamment des représentantes et des représentants d'autres ministères et des dirigeantes historiques du mouvement féministe) ainsi que des consultations continues avec le milieu associatif plus large. Selon une ancienne fonctionnaire du MCFDF, les relations de confiance qu'elles avaient établies avec les associations féministes et avec les bailleurs de fonds étaient cruciales pour assurer cette collaboration: "Qui tu étais à titre personnel et qui tu connaissais pouvait directement influencer les résultats politiques » (Champ 2018: 51; notre traduction). C'est sur cette base qu'elles ont élaboré la Politique EFH et son plan d'action de 2012 à 2014 .

Cette approche collaborative diffère profondément de l'approche du cluster violence postséisme, car elle a permis l'élaboration de la Politique EFH et de son plan d'action de façon techniquement rigoureuse avec la participation de maintes associations des femmes à Port-au-Prince et à l'échelle provinciale, sans compromettre la direction politique formellement portée par l'État haïtien (République d'Haïti 2014a et 2014b). Par contre, selon une ancienne cadre du MCFDF, la participation du MCFDF et d'autres ministères restait largement ancrée, à cette étape, dans des engagements individuels plutôt que dans un réel engagement institutionnel (Champ 2018).

\section{La Politique EFH et son plan d'action}

La Politique EFH envisage la mise en œuvre d'un ensemble de mesures structurantes sur un horizon de 20 ans (de 2014 à 2034), pour éliminer les discriminations et promouvoir l'égalité entre les femmes et les hommes dans les domaines clés de la vie nationale haïtienne. Le premier chapitre reprend l'état des lieux présenté dans le Diagnostic. Le deuxième expose les fondements juridiques, politiques et autres, ainsi que les principes directeurs de la Politique EFH, soit l'égalité, l'équité, la non-discrimination, l'inclusion sociale, la cohérence et la transparence. Ce chapitre explique aussi l'importance du partenariat (entre l'État, la société civile, le secteur privé, le secteur universitaire, les partis politiques, les collectivités territoriales et les PTF) comme pierre angulaire de la mise en œuvre des engagements : «La présente politique, porteuse d'une vision partagée, suppose de créer les conditions pour que toutes les institutions de la société participent à la modification intégrale de la division sexuée des rôles et des responsabilités » (République d'Haïti 2014b : 81). 
Les éléments plus opérationnels se trouvent dans les derniers chapitres. Le troisième chapitre présente les objectifs et les mesures envisagées dans chaque domaine. Par exemple, en matière d'égalité de droit et de justice équitable, la Politique EFH mentionne des mesures pour modifier des lois discriminatoires à l'égard des femmes, promouvoir de nouvelles lois qui assurent l'égalité et la protection contre la violence, et former les acteurs et les actrices du système en vue d'assurer la pleine application de ces lois. Ce chapitre contient aussi des engagements en matière de santé maternelle, sexuelle et reproductive, d'éducation, d'égalité économique et de participation égalitaire des femmes aux instances dirigeantes.

C'est dans le quatrième et dernier chapitre que figurent les dispositions pour assurer le suivi de la Politique EFH. En particulier, l'État s'est engagé à établir et à appuyer une structure interministérielle de pilotage, à mobiliser un ensemble d'outils de gestion axée sur les résultats, à assurer la budgétisation sensible au genre, à faire l'évaluation périodique de la mise en œuvre de la Politique EFH et à rendre des comptes régulièrement à cet égard. Loin d'être un exercice purement technique, cette démarche est présentée comme un levier pour susciter l'engagement social requis afin d'assurer les transformations majeures (et dont la contestation est donc prévisible) envisagées dans la Politique EFH : «Cet outil concret, issu d'un travail collégial impliquant la participation d'acteurs, d'actrices des différentes divisions administratives du pays, doit amener à la construction d'une société démocratique et inclusive » (République d'Haïti 2014b : 82).

Le dernier chapitre de la Politique EFH est aussi la passerelle qui mène au Plan national EFH, lequel codifie des engagements plus spécifiques pour la mise en œuvre de la Politique EFH pendant ses six premières années d'existence. Il contient des engagements budgétaires, soit que, en plus du budget accordé au MCFDF (qui équivaut environ à $1 \%$ du budget national), les autres ministères visés consacreront $1 \%$ de leur budget à la réalisation des mesures d'EFH dans leur secteur pour la première année et que ce montant pourrait être augmenté sur la base d'un exercice annuel de budgétisation sensible au genre (République d'Haïti 2014a : 3).

Pour chaque objectif, le Plan d'action EFH indique les mesures concrètes, les indicateurs de suivi et l'institution responsable. Par exemple, pour l'objectif trois, qui consiste à promouvoir des valeurs égalitaires et la non-discrimination dans le système éducatif, le Plan d'action EFH codifie 11 mesures, y compris la suivante : " Mettre en place un programme de formation pour les auteur-e-s, graphistes, directeurs et directrices d'écoles et maisons d'édition sur les stéréotypes dans les manuels scolaires » pour promouvoir l'EFH dans les écoles (République d'Haïti 2014a : 6-7). Le ministère de l'Éducation nationale et de la Formation professionnelle (MENFP) est chargé de diriger l'application de ces mesures. Par rapport à la gouvernance et au suivi, le Plan d'action EFH compte sept mesures, notamment les suivantes: «Présenter un rapport annuel d'application des mesures l'EFH» (ibid. : 25-26), renforcer le partenariat entre les membres de la Table thématique genre et « Mettre en 
LA POLITIQUE D'ÉGALITÉ FEMMES HOMMES 2014-2034 EN HAÏTI | 119

place un observatoire de l'égalité femmes hommes » (ibid. : 26). Le MCFDF doit diriger le suivi de ces mesures.

L'approbation officielle de ces documents, à la fin de l'année 2014, a eu lieu discrètement. Pour assurer leur visibilité, le MCFDF et ses partenaires alliés ont profité de deux moments clés pour faire connaître ces documents à un public plus large. La Politique EFH a été présentée le 8 mars 2015, à la célébration officielle de la Journée internationale des femmes à Port-au-Prince. Quant au Plan d'action EFH, il a été dévoilé au public le 28 mai 2015, à la célébration de la Journée internationale de la santé de la femme. En plus de la ministre à la Condition féminine et aux Droits des femmes, le président Martelly et plusieurs dignitaires, y compris l'ambassadrice du Canada, ont pris la parole pour appuyer ces acquis, ce qui a généré une large couverture médiatique (Le Nouvelliste 2015; Champ 2018).

La construction collective de la Politique EFH et son lancement public au début de 2015 reflètent bien le "génie stratégique » du mouvement des Haïtiennes mentionné par Côté (2014a), soit la capacité des fémocrates et du mouvement social de créer ou de saisir d'importantes occasions inscrites dans les calendriers institutionnels. Ils reflètent leur capacité de mobiliser des appuis techniques, financiers et même politiques des partenaires internationaux. Ils démontrent que des acteurs institutionnels peu connus pour leurs pratiques féministes, tel l'ancien président Martelly, peuvent se joindre au jeu lorsque cela leur convient. Par contre, ces événements et leur suite moins heureuse révèlent aussi les (f)acteurs institutionnels et sociaux qui contraignent l'application des engagements dans ce domaine.

\section{La mise en œuvre limitée des acquis politiques}

Dans des circonstances idéales, le gouvernement aurait lancé la mise en œuvre du Plan d'action EFH pendant la dernière année de son mandat. Cependant, les années électorales sont souvent tumultueuses en Haïti et 2015 est entrée dans ce cadre, avec des scrutins contestés en août et en octobre 2015, la crise de gouvernance qui en a découlé et le départ peu cérémonieux du président Martelly en février 2016. Le gouvernement de transition a organisé les scrutins qui ont mené à l'élection du président Moïse et à l'entrée en fonction du premier ministre Lafontant au début de 2017, mais il n'a pu freiner la crise macroéconomique ou ses retombées fiscales fort négatives pour les institutions publiques (SGNU 2016 et 2017).

Dans ce contexte très contraignant, le MCFDF peinait à gérer les affaires courantes. Il a participé aux processus d'élaboration du budget en 2016, mais il n'est pas arrivé à assurer l'adoption d'un budget sensible au genre. Tout simplement, il a continué à fournir des services essentiels dans les départements et à assurer une représentation minimale dans les instances internationales. Dans un rapport soumis au comité de l'Organisation des Nations unies (ONU) chargé du suivi de la CEDEF, le MCFDF a suggéré que, malgré les contraintes susmentionnées, Haïti avançait dans la 
mise en œuvre de la Politique EFH (MCFDF 2016). Par exemple, il a lancé une nouvelle campagne de sensibilisation pour le respect des corps des filles et des femmes en mars 2016. Il a collaboré avec le ministère de la Justice pour renforcer les bureaux d'assistance légale dans certains départements, notamment leur capacité de prendre en charge les femmes victimes de violence. Il a contribué à l'élaboration du nouveau Code pénal, élaboré la Loi sur la prévention et la sanction des violences faites aux femmes et appuyé le plaidoyer pour assurer leur approbation par le Parlement. En outre, le MCFDF a aidé à la campagne menée pour augmenter la représentation des femmes au Parlement par l'entremise des élections en 2015, mais la directrice générale du MCFDF a souligné qu'elle « ne [pouvait] s'empêcher [de] déplorer le fait qu'aucune femme n'ait été élue au Parlement » malgré ces efforts (MCFDF 2016 : 8). Les contraintes découlant de la crise politique et de ses séquelles économiques ont donc limité énormément la capacité du MCFDF à assurer la mise en œuvre initiale du Plan d'action EFH.

C'est aussi dans cette conjoncture que les associations des femmes vivant en situation d'handicap ont fait irruption sur la scène nationale. En mai 2016, l'Association de filles et femmes au soleil (AFAS) et l'Union des femmes à mobilité réduite d'Haïti (UFMORH) ont publié un rapport dénonçant leur exclusion des consultations qui avaient mené à l'élaboration de la Politique EFH. Elles critiquaient l'absence d'attention, dans cette politique, aux besoins spécifiques des filles et des femmes handicapées. Elles exigeaient la modification de la Politique EFH et de son plan d'action pour pallier ces lacunes (BSEIPH 2016). Comme l'ont expliqué les dirigeantes de ces organisations, ces demandes étaient le fruit d'un travail assidu d'analyse et de plaidoyer, appuyé par le Disability Rights Fund, au cours des années antérieures. Elles étaient aussi provoquées par la posture plutôt universaliste des dirigeantes féministes historiques, à l'égard des femmes vivant en situation d'handicap. Selon les paroles d'une activiste handicapée, ces organisations ne tiennent pas compte de la réalité des femmes handicapées: "C'est seulement pour "les femmes " en général, pas pour les femmes handicapées. Et elles travaillent seulement pour les femmes de Port-au-Prince » (Entretien 2018a).

L'établissement d'un nouveau gouvernement, en février et en mars 2017, a présenté des occasions pour relancer la Politique EFH. En mars, la première dame, Martine Moïse, a invité, avec l'appui d'ONU-Femmes, des personnalités du nouveau gouvernement, du mouvement des femmes et de la coopération internationale à un souper-causerie. Par la suite, la nouvelle ministre à la Condition féminine et aux Droits des femmes, Eunide Innocent, a relancé la collaboration interministérielle pour assurer la mise en œuvre de la Politique EFH. Les cadres du MCFDF ont persévéré avec obstination pour obtenir l'aval du premier ministre : après beaucoup de pressions, il a envoyé, en août, une lettre à tous les ministères visés, leur rappelant leurs obligations dans ce dossier et les enjoignant à participer à des ateliers interministériels de sensibilisation (Champ 2018). 
Sur cette base, le MCFDF a organisé deux ateliers interministériels en août et en septembre 2017. Selon des cadres du MCFDF, l'atelier tenu avec les hauts responsables politiques de huit ministères n'a pas été un succès, car ni le premier ministre ni d'autres ministres clés n'étaient présents. Par contre, l'atelier réunissant les cadres de ces entités a occasionné de meilleurs échanges et des engagements de suivi : d'abord, pour établir les unités genre dans chaque ministère et, ensuite, pour créer le comité interministériel EFH et son secrétariat afin d'assurer le suivi coordonné des engagements inscrits dans la Politique EFH.

En février 2018, et ce, malgré le gel de son budget, le MCFDF a lancé une campagne de sensibilisation en Jérémie pour présenter la Politique EFH et son plan d'action. C'est aussi dans ce contexte que la ministre et les cadres du MCFDF ont rencontré leurs homologues au Bureau du secrétaire d'État à l'intégration des personnes handicapées, pour voir comment il leur serait possible de répondre aux demandes d'inclusion des associations des femmes vivant en situation d'handicap. Des rencontres ont été organisées avec l'AFAS et l'UFMORH. Par la suite, la ministre a décidé que l'éventuelle mise à jour du Plan d'action EFH devrait refléter les intérêts de ce sous-secteur et que les associations devraient être consultées à cet égard (Entretien 2018b).

Pendant ce temps, certains partenaires internationaux de l'EHF continuaient à chercher des portes d'entrée pour promouvoir le suivi de la Politique EFH et de son plan d'action. En juin 2017, l'ambassade du Canada et ONU-Femmes convoquaient le Groupe de travail des partenaires en EHF, mais sans la participation du MCFDF ou des instances associatives haïtiennes, donc sans mobiliser la Table thématique genre conçue pour assurer la coordination interinstitutionnelle (Champ 2018). À la suite de la présentation d'une politique féministe d'assistance internationale en 2017, le Gouvernement du Canada a annoncé de nouveaux appuis aux organisations locales de femmes. Dans ce contexte, le Centre d'étude et de coopération internationale (CECI) a lancé en 2018 le programme Voix et leadership des femmes en Haïti, une initiative de 8 millions de dollars canadiens qui appuiera 30 organisations de défense des droits des femmes et des filles, en partenariat avec le MCFDF (CECI 2019).

Donc, en dépit de l'appui politique limité au plus haut niveau ainsi que d'importantes contraintes budgétaires, le MCFDF et ses partenaires ont pris l'initiative de relancer la mise en œuvre de la Politique EFH et de son plan d'action, et ont réussi à le faire. Cependant, l'augmentation dramatique du prix du carburant et les émeutes du 6 juillet 2018, suivies de la démission du gouvernement Lafontant dans un contexte de crise fiscale aiguë, ont jeté de l'eau glacée sur les ambitions de ce réseau. Dans cette conjoncture extrêmement contraignante (Baranyi 2019), il n'est pas surprenant d'observer le peu de progrès, en termes pratiques, dans la plupart de ces dossiers, depuis le changement du gouvernement en mai 2018. Ni le nouveau Code pénal ni la Loi sur les violences faites aux femmes n'ont été ratifiées par le Parlement. La mise en œuvre de la Loi sur la filiation, la maternité et la paternité tarde toujours (Lecarpentier 2018). Aucun budget n'ayant été adopté, l'idée de proposer un budget 
BARANYI ET CHAMP $\mid 122$

sensible au genre est restée lettre morte. Les unités genre des autres ministères et le comité interministériel de suivi n'ont pas été activés. Aucun rapport officiel sur la mise en œuvre de la Politique EFH et de son plan d'action n'a été présenté au Parlement ou au public, même si un rapport partiel, sur le volet de l'accès à la justice, est en cours d'élaboration (MCFDF à paraître). À la fin de 2018, ce manque de progrès a amené Kay Famn à déclarer que l'État «ne montre pas une volonté de mettre efficacement en œuvre la politique 2014-2034 d'égalité homme-femme et le plan national 2017-2021 [sic] de lutte contre les violences faites aux femmes » (Alterpresse 2018).

Dans ce contexte, l'analyse de Sincimat Fleurant (2018), selon laquelle il n'y a pas encore eu d'adoption réelle ou de routinisation bureaucratique de l'approche genre au-delà du MCFDF, reste fort pertinente. Cette faible institutionnalisation est aussi liée au roulement du personnel d'expérience au MCFDF. Voici ce qu'a exprimé une ancienne fonctionnaire du MCFDF :

Maintenant [...] nous n'avons pas les mêmes acteurs : les personnes qui étaient impliquées pendant la période de l'élaboration [de la Politique EFH] ne sont pas les mêmes à l'étape de l'application. Nous avons aussi perdu des personnalités clés pendant le séisme. Donc là nous devons travailler avec des nouveaux acteurs qui ont besoin de temps pour apprendre et pour devenir motivés. Cela retarde aussi le processus de mise en œuvre (Champ 2018 : 80; notre traduction).

Néanmoins, le problème va bien au-delà du manque d'institutionnalisation bureaucratique. L'écart entre les engagements politiques et les pratiques quotidiennes des institutions révèle l'ampleur des contraintes institutionnelles, structurelles et culturelles qui compliquent la transformation de relations genrées en Haïti, et ce, en dépit de l'agentivité ardue des fémocrates, du mouvement féministe et de leurs partenaires internationaux.

\section{Conclusion}

Cet arbre de l'égalité des genres, que nous avons planté voilà quelques décennies, est exactement à l'image de ce jeune chêne : sa partie visible semble bien épanouie, mais ses racines ne sont pas aussi profondes que certains le pensent. Et il est bien connu, ma petite, que les arbres qui ont moins de racines que de branches sont souvent à la merci de ces grands vents qu'on croyait disparus [...].

Récit d'une grand-mère sénégalaise à sa petite-fille, relaté par Boucar Diouf $(2015: 28)$

L'histoire de la Politique EFH, interprétée par l'entremise d'un cadre théorique féministe institutionnaliste, laisse bien voir la dialectique de l'agentivité 
féministe et des contraintes récurrentes dans le contexte haïtien. Elle capte d'abord le génie stratégique du mouvement féministe, l'importance des alliances entre les militantes de la société civile, les fémocrates travaillant au sein de l'État (notamment du MCFDF) et leurs partenaires de la coopération internationale, ainsi que leur capacité de mobiliser des ressources de façon audacieuse pour créer des occasions ou profiter de celles qui sont offertes par l'État national ainsi que par les normes et la coopération internationales. Cette assemblage diffère des dynamiques vécues après le séisme de 2010, marquées par la dominance des acteurs internationaux, de paradigmes et de mécanismes d'action humanitaire critiqués par les féministes postcoloniales. L'histoire de la Politique EFH reflète plutôt un retour à une coopération plus équilibrée, sous la direction stratégique des parties prenantes nationales.

Par contre, notre lecture féministe institutionnaliste de la faible mise en œuvre de la Politique EFH et de son plan d'action depuis 2014 illustre aussi la récurrence des contraintes institutionnelles au sens large : depuis les capacités et les ressources limitées des fémocrates du MCFDF et des microrésistances bureaucratiques dans les autres ministères, en passant par l'attitude ambiguë des autorités sous plusieurs gouvernements, les crises de gouvernance politique récurrentes, les contraintes fiscales aggravées par la crise économique depuis 2016 et, bien sûr, les contraintes patriarcales plus profondes mentionnées par maintes auteures. Dans le contexte de ces lourdes contraintes endogènes, les incohérences récentes des partenaires internationaux apparaissent comme des obstacles plutôt mineurs, malgré les dynamiques postséisme soulignées à juste titre par les critiques postcoloniales.

Notre analyse a aussi fait ressortir une dialectique dans la dialectique, soit la contestation, par les femmes vivant en situation d'handicap, de leur exclusion des processus qui ont mené à l'adoption de la Politique EFH. Cette contestation rappelle la difficulté de mettre en œuvre les principes de l'inclusion intersectionnelle, même dans les espaces féministes, en dépit des orientations stratégiques suggérées par des dirigeantes historiques comme Merlet (2010). Par contre, l'engagement du MCFDF d'inclure ces associations dans la mise à jour du Plan d'action national EFH ouvre la porte à un changement des pratiques établies.

Notre étude propose également des pistes pour l'action collective en Haïti. Elle souligne l'importance de continuer à articuler les ressources du MCFDF, du mouvement des femmes et l'appui des partenaires internationaux traditionnels comme ONU-Femmes et le Canada. Ensemble, ces partenaires pourraient maintenir le cap sur la mise en œuvre de la Politique EFH et de son plan d'action, malgré les contraintes notées ci-dessus. Elles pourraient continuer à créer des occasions pour faire valoir les engagements de l'État dans les processus budgétaires nationaux et dans le suivi du Plan d'action EFH avant qu'il prenne fin en 2020. En plus de ces démarches prévisibles, il serait intéressant d'ouvrir la porte à de nouvelles alliances intersectionnelles, notamment avec le secteur des femmes vivant en situation d'handicap, compte tenu de la passerelle que cela pourrait élargir avec l'important 
mouvement des personnes vivant dans cette situation. La rédaction du prochain plan d'action EFH offrira peut-être une possibilité à cet égard.

Notre étude indique en outre des pistes de recherche empirique et de réflexion théorique. Il est crucial de continuer la recherche lancée par Sincimat Fleurant, sur la mise en œuvre de l'approche genre dans les ministères autres que le MCFDF. La publication espérée du rapport du MCFDF (à paraître), sur le volet accès à la justice, pourrait offrir des pistes à ce sujet. Les alliances entre les institutions historiques du mouvement féministe (à l'échelle de la société et de l'État) et les femmes vivant en situation d'handicap, souvent plus jeunes que les dirigeantes historiques, méritent aussi un suivi scientifique. Ces actrices arriveront-elles à négocier une coalition au cours des prochaines années ou resteront-elles piégées dans des pratiques d'inclusion sans substance, comme l'a mentionné une dirigeante du secteur (Entretien 2018a)?

Finalement, il serait intéressant d'approfondir la comparaison de la mise en œuvre des politiques EFH en Haïti et dans des pays antillais comme la Jamaïque, abordées dans Hosein et Parpart (2016). Un cadre théorique féministe institutionnaliste pourrait contribuer à l'analyse de ces enjeux et au dialogue avec d'autres perspectives féministes, notamment sur les possibilités d'élargir l'agentivité hétérogène (soit les racines de l'arbre féministe dont parle la grand-mère sénégalaise) dans un contexte de résistances patriarcales et de crises récurrentes dans ces pays.

\section{RÉFÉRENCES}

ALTERPRESSE

2018 «L'organisation Kay Famn critique l'indifférence de l'État face aux disparités de genre », AlterPresse, [En ligne], [www.alterpresse.org/spip.php? article23889\#.XHWFXIhKg2w] (4 juillet 2019).

2011 «Un quota de 30 pour cent, préconise l'organisation "Fanm Yo La" ", AlterPresse, [En ligne], [www.alterpresse.org/spip.php?article11507] (10 juillet 2017).

BARANYI, Stephen

2019 «Haïti : le chaos, la refonte du système ou le bricolage continu? », Diplomatie, 100, $1:$ 30-35.

BARANYI, Stephen (dir.)

2008 The Paradoxes of Peacebuilding Post-9/11. Montréal/Kingston, Vancouver, University of British Columbia Press.

BARANYI, Stephen, et Ilionor LOUIS

2016 «(Dis)ability and Development in Haiti: Beyond One-dimensional Views », Third World Thematics, 1, 3:1-14.

BERENI, Laure, et Anne REVILLARD

2012 «Un mouvement social paradigmatique? Ce que le mouvement des femmes fait à la sociologie des mouvements sociaux », Sociétés contemporaines, 1, $85: 17-41$. 
LA POLITIQUE D’ÉGALITÉ FEMMES HOMMES 2014-2034 EN HAÏTI | 125

BILGE, Sirma

2010 «De l'analogie à l'articulation: théoriser la différenciation sociale et l'inégalité complexe », L'Homme et la société, 2, 176-177: 43-64.

BUREAU DU SECRÉTAIRE D'ÉTAT À L'INTÉGRATION DES PERSONNES HANDICAPÉES (BSEIPH)

2016 «L'AFAS et l'UFMORH contre les discriminations à l'égard des femmes », BSEIPH, [En ligne], [www.seiph.gouv.ht/lafas-et-lufmorh-contre-lesdiscriminations-a-legard-des-femmes/] (11 mai 2016).

CENTRE D'ÉTUDE ET DE COOPÉRATION INTERNATIONALE (CECI)

2019 «Voix et leadership des femmes en Haïti », CECI, [En ligne], [ceci.ca/fr/pro jets/voix-et-leadership-des-femmes-en-haiti-vlf-haiti] (12 septembre 2019).

CHAMP, Hannah

2018 A Critical Feminist Institutional Analysis of Haiti's 'Politique d'égalité femmes hommes '. Thèse de maîtrise. Ottawa, Université d'Ottawa.

CHARLES, Ketleine

2018 «Quelques réflexions sur l'application du quota en Haïti », dans Sabine Lamour, Denyse Côté et Darline Alexis (dir.), Déjouer le silence. Contrediscours sur les femmes haïtiennes. Montréal, Les éditions du remue-ménage/ PressuniQ/Mémoire d'encrier : 160-172.

CÔTÉ, Denyse

2014a «'Anpil fanm tonbe, nap kontinye vanse': luttes féministes en Haïti », Possibles, 38, 1 : 209-223.

$2014 \mathrm{~b}$ «Un espace politique menacé : le mouvement féministe haïtien et les effets pervers de l'aide humanitaire », dans Nancy Thede et Mélanie DufourPoirier (dir.), L'Amérique latine : laboratoire du «politique » autrement. Montréal, Presses de l'Université de Québec : 123-140.

DIOUF, Boucar

2015 Rendez à ces arbres ce qui appartient à ces arbres. Montréal, Les éditions de La Presse.

DUFFIELD, Mark

2007 Development, Security and Unending War: Governing the World of Peoples. Oxford, Polity Press.

DUMONT, Marie

2017 Corruption and Women in Politics: Correlation, Institutional Context, or Coincidence? Thèse de maîtrise. Ottawa, Université d'Ottawa.

EYBEN, Rosalind, et Laura TURQUET (dir.)

2013 Feminists in Development Organizations: Change from the Margins. Rugby, Practical Action Publishing.

HOSEIN, Gabrielle J., et Jane PARPART (dir.)

2016 Negotiating Gender Policies in the Caribbean. Feminist Strategies, Masculinist Resistance and Transformational Possibilities. Londres/New York, Rowman \& Littlefield. 
JAD, Islah

2007 «NGOs: Between Buzzwords and Social Movements », Development in Practice, 17, 4-5 : 622-629.

LAMOUR, Sabine, Denyse CÔTÉ et Darline ALEXIS (dir.)

2018 Déjouer le silence. Contre-discours sur les femmes haïtiennes. Montréal, Les éditions du remue-ménage/PressuniQ/Mémoire d'Encrier.

LEBON, Nathalie

2010 «Introduction », dans Elizabeth Maier et Nathalie Lebon (dir.), Women's Activism in Latin America and the Caribbean. Engendering Social Justice, Democratizing Citizenship. New Brunswick (NJ), Rutgers University Press : 3-25.

LECARPENTIER, Pauline

2018 «L'évolution du droit de la filiation et la réforme sur la 'paternité responsable' en Haïti », dans Sabine Lamour, Denyse Côté et Darline Alexis (dir.), Déjouer le silence. Contre-discours sur les femmes haïtiennes. Montréal, Les éditions du remue-ménage/PressuniQ/Mémoire d'encrier : 263-276.

LE NOUVELLISTE

2015 «Vers la matérialisation de l'égalité de genre », Le Nouvelliste, [En ligne], [lenouvelliste.com/lenouvelliste/article/145523/Vers-la-materialisation-delegalite-de-genre] (31 mai 2015).

MACKAY, Fiona, Meryl KENNY et Louise CHAPPELL

2010 «New Institutionalism through a Gender Lens: Towards a Feminist Institutionalism? », International Political Science Review, 31, 5 : 573-588.

MACKAY, Fiona, et Mona Lena KROOK

2015 Gender, Politics and Institutions: Towards a Feminist Institutionalism. Basingstoke, Palgrave Macmillan.

MAGLOIRE, Danièle

2018 «L'antiféminisme en Haïti », dans Sabine Lamour, Denyse Côté et Darline Alexis (dir.), Déjouer le silence. Contre-discours sur les femmes haïtiennes. Montréal, Les éditions du remue-ménage/PressuniQ/Mémoire d'encrier : 199-212.

MAIER, Elizabeth

2010 "Concluding Reflections », dans Elizabeth Maier et Nathalie Lebon (dir.), Women's Activism in Latin America and the Caribbean. Engendering Social Justice, Democratizing Citizenship. New Brunswick (NJ), Rutgers University Press : 337-352.

MAIER, Elizabeth, et Nathalie LEBON (dir.)

2010 Women's Activism in Latin America and the Caribbean. Engendering Social Justice, Democratizing Citizenship. New Brunswick (NJ), Rutgers University Press. 
LA POLITIQUE D'ÉGALITÉ FEMMES HOMMES 2014-2034 EN HAÏTI | 127

MAZUR, Amy G.

2009 «Les mouvements féministes et l'élaboration des politiques dans une perspective comparative », Revue française de science politique, 59, $2: 325$ 351.

MCFALLS, Laurence

2010 «Benevolent Dictatorship: The Formal Logic of Humanitarian Government », dans Didier Fassin et Mariella Pandolfi (dir.), Contemporary States of Emergency: The Politics of Military and Humanitarian Intervention. New York, Zone : 317-333.

MCFEE, Deborah

2016 «Narratives, the State and National Gender Policies in the Anglophone Caribbean », dans Gabrielle J. Hosein et Jane Parpart (dir.), Negotiating Gender Policies in the Caribbean. Feminist Strategies, Masculinist Resistance and Transformational Possibilities. Londres/New York, Rowman \& Littlefield : 109-129.

MERLET, Myriam

2010 "Haiti: Women in the Conquest of Full Citizenship in an Endless Transition ", dans Elizabeth Maier et Nathalie Lebon (dir.), Women's Activism in Latin America and the Caribbean. Engendering Social Justice, Democratizing Citizenship. New Brunswick (NJ), Rutgers University Press : 129-139.

MINISTÈRE À LA CONDITION FÉMININE ET AUX DROITS DES FEMMES (MCFDF)

À paraître Rapport d'évaluation de la mise en œuvre de l'orientation Égalité de droit et justice équitable entre les femmes et les hommes du PANEFH 2014-2020. Port-au-Prince, Gouvernement d'Haïti.

2016 Discours de la Directrice générale. 63e session du Comité pour l'élimination de la discrimination à l'égard des femmes. Genève, Nations unies.

2013 Diagnostic des inégalités de genre. Port-au-Prince, Gouvernement d'Haïti.

2008 Application de la Convention pour l'élimination de toutes les formes de discriminations à l'égard des femmes (CEDEF). Rapports combinés 19822006. Port-au-Prince, Gouvernement d'Haïti.

PECK, Raoul

2013 Assistance mortelle, [Enregistrement vidéo], ARTE France, Velvet Film, Figuier Production, RTBF et Entre Chien et Loup, HD Cam, 100 min.

RÉPUBLIQUE D'HAÏTI

2014a Plan d'action national d'égalité femmes hommes 2014-2020. Port-au-Prince, Gouvernement d'Haïti.

2014b Politique d'égalité femmes hommes 2014-2034. Port-au-Prince, Gouvernement d'Haïti. 
BARANYI ET CHAMP | 128

RÉPUBLIQUE D'HAÏTI ET ORGANISATION DE COOPÉRATION ET DE DÉVELOPPEMENT ÉCONOMIQUES (OCDE)

2011 Rapport 2011 sur l'engagement international dans les États fragiles. Paris/ Port-au-Prince, OCDE/Gouvernement d'Haïti.

ROMULUS, Célia

2018 «Le potentiel d'une recherche féministe décoloniale pour déconstruire le récit de l'État fragile haïtien », dans Sabine Lamour, Denyse Côté et Darline Alexis (dir.), Déjouer le silence. Contre-discours sur les femmes haïtiennes. Montréal, Les éditions du remue-ménage/PressuniQ/Mémoire d'encrier : 3554.

SECRÉTAIRE GÉNÉRAL DES NATIONS UNIES (SGNU)

2017 Rapport du secrétaire général sur la mission des Nations unies pour la stabilisation en Haïti. Port-au-Prince, Nations unies Haïti.

2016 Rapport du secrétaire général sur la mission des Nations unies pour la stabilisation en Haïti. Port-au-Prince, Nations unies Haïti.

SINCIMAT FLEURANT, Rose Esther

2018 «Pratiques institutionnelles et représentations sociales du genre dans l'administration publique haïtienne, 2013-2015 », dans Sabine Lamour, Denyse Côté et Darline Alexis (dir.), Déjouer le silence. Contre-discours sur les femmes haïtiennes. Montréal, Les éditions du remue-ménage/PressuniQ/ Mémoire d'encrier : 147-159.

THAME, Maziki, et Dhanaraj THAKUR

2016 «The National Policy on Gender Equality in Jamaica. (En)gendering Equity in Neo-liberal Times ", dans Gabrielle J. Hosein et Jane Parpart (dir.), Negotiating Gender Policies in the Caribbean. Feminist Strategies, Masculinist Resistance and Transformational Possibilities. Londres/New York, Rowman \& Littlefield : 153-172.

VERSCHUUR, Christine

2010 «Diversité des mouvements de femmes, des pratiques et des théories féministes: la contribution des études de genre aux études de développement », Genre, colonialisme et diversité des mouvements de femmes. Cahiers genre et développement, 7 : 15-24.

Entretiens additionnels (aux 19 entretiens effectués par Hannah Champ en 2017)

2018a Entretien avec des représentantes de l'Union des femmes à mobilité réduite d'Haïti (UFMORH), 29 juin, Port-au-Prince.

2018b Entretien avec la ministre à la Condition féminine et aux Droits des femmes, 3 juillet, Port-au-Prince. 